\title{
Experimental Study of Contact Transition Control Incorporating Joint Acceleration Feedback
}

\author{
W. L. Xu, Senior Member, IEEE, J. D. Han, and S. K. Tso, Senior Member, IEEE
}

\begin{abstract}
Joint acceleration and velocity feedbacks are incorporated into a classical internal force control of a robot in contact with the environment. This is intended to achieve a robust contact transition and force tracking performance for varying unknown environments, without any need of adjusting the controller parameters. A unified control structure is proposed for free motion, contact transition, and constrained motion in view of the consumption of the initial kinetic energy generated by a nonzero impact velocity. The influence of the velocity and acceleration feedbacks, which are introduced especially for suppressing the transition oscillation, on the postcontact tracking performance is discussed. Extensive experiments are conducted on the third joint of a three-link direct-drive robot to verify the proposed scheme for environments of various stiffnesses, including elastic (sponge), less elastic (cardboard), and hard (steel plate) surfaces. Results are compared with those obtained by the transition control scheme without the acceleration feedback. The ability of the proposed control scheme in resisting the force disturbance during the postcontact period is also experimentally investigated.
\end{abstract}

Index Terms-Acceleration feedback, contact transition, force tracking control, robot, velocity feedback.

\section{INTRODUCTION}

$\mathbf{T}$ HE problem of controlling robots in contact with objects is of central importance in many applications. Robots are subjected to interaction forces whenever they perform tasks involving motion that is constrained by the environment, such as precision assembly, grinding, finishing, deburring, and so forth. In such motions, the interaction forces must be accommodated rather than suppressed to comply with the environmental constraints. The robot force control can be divided into three phases, i.e., free motion, contact transition, and force tracking phases. Large impact force and bouncing may occur during the contact transition, while disturbances may result in the loss of contact during the force tracking phase. Thus, a robust contact transition and force tracking control in an unknown or changing environment has been pursued.

Mills and Cokhorst [1]-[3] developed a discontinuous controller to deal with the contact instability problem and tackle events of contact loss and trajectory tracking. Volpe and Khosla

Manuscript received May 8, 1998; revised July 13, 1999. Recommended by Technical Editor T. J. Tarn

W. L. Xu is with the Institute of Technology and Engineering, Massey University, Palmerston North, New Zealand.

J. D. Han is with the Robotics Laboratory, Shenyang Institute of Automation, Chinese Academy of Sciences, Shenyang 110015, China.

S. K. Tso is with the Center for Intelligent Design, Manufacturing and Automation, City University of Hong Kong, Hong Kong.

Publisher Item Identifier S 1083-4435(00)07830-3.
[4] also proposed a discontinuous approach for adjusting force control gains during impact to achieve stable bounce-free contact transitions in hard-to-hard contact situations. Hogan [5] implemented impedance control in experiments involving contact transitions and achieved stability against a stiff environment, while Vossoughi and Donath [6] employed impedance methods for environments with varying stiffnesses. Serajia et al. [7], [8] integrated hybrid position/force control with impedance control for employing force feedback to enable the robot to achieve a desired contact force. Youcef-Toumi and Guts [9] developed a dimensionless representation of impact behavior and used integral force compensation with velocity feedback to improve impact response, while Khatib and Burdick [10] presented a method for dissipating impact oscillations by increasing the velocity gains of a proportional-derivative force controller. Qian and De Schutter [11] presented an active nonlinear damping approach by examining the force signal derivative. Hyde and Cutkosky [12], [13] proposed an input command preshaping method by modifying feedforward information to minimize the contact oscillations.

The above proposed control laws, such as the discontinuous control [1]-[4], the impedance control [5]-[10], the active damping approach [11], the input command preshaping [12], [13], succeeded in stabilizing the impact event with respect to specific environments. However, these algorithms are dependent on environment dynamics and require the environment to be accurately modeled. Hence, the parameters in these control laws have to be redesigned if the contacted environment differs. For dealing with uncertainties in different task environments, Vukobratovic [14] introduced environmental dynamics into a dynamically interactive control scheme. Weng and Young [15] proposed an adaptive fuzzy law for transition control based on the identification of the contacted environment. When the environment is stiff, such identification is impossible to be completed because the time interval between the impact occurring and the contact force reaching its desired value is very short. To tackle three different types of control problems (free motion, impact, and postcontact), Mandal and Payandeh [16] proposed a unified control proportional-integral-derivative (PID) force controller incorporating a knowledge base for tuning the gains involved. However, the knowledge base is quite coarse and unable to provide a good estimate due to the noise in the force sensor.

It is essential for many industrial tasks that the robot avoids significant bouncing and maintains contact with an environment during the contact transition. This requires that the initial 


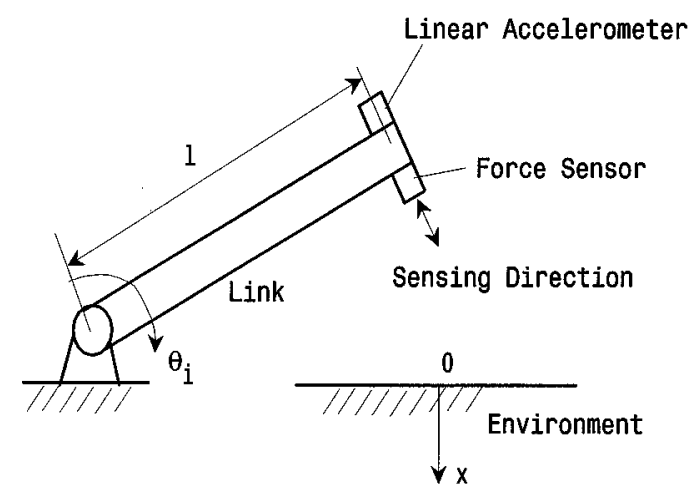

Fig. 1. Single-link robot.

kinetic energy that is generated by the nonzero impact velocity be dissipated within a short time period. Velocity feedback helps consume the energy and stabilize the contact transition theoretically [9], [10], [13]. However, in practice, especially when the environment is less elastic, this approach is hardly effective due to the limited bandwidth of the velocity closed loop. To remedy this problem in hard contact, a joint acceleration feedback control has been employed, since its bandwidth is wide enough in response to the rapid change of force. Liu [17] made use of the acceleration feedback control as an inner loop to reduce the force tracking error during the postcontact phase. Tarn, et al. [18], [19] proposed a positive acceleration feedback control and an event-driven switching scheme to control the impact force and bouncing during the contact transition. In this paper, a joint acceleration feedback is introduced into a classical integral force controller (with a velocity feedback). This aims to achieve a robust contact transition and force tracking without adjusting the controller parameters for various unknown or changing environments. The proposed control scheme is discussed in view of that the joint acceleration feedback helps a joint actuator dissipate initial energy when a contact is established. Extensive experiments are conducted on the third joint of a three-link direct-drive robot to verify the proposed scheme for various environments in contact, including elastic (sponge), less elastic (cardboard), and hard (steel plate) surfaces. Results are compared with those obtained by the transition control without acceleration feedback.

\section{CONTACT TRANSITION CONTROL INCORPORATING VELOCITY AND ACCELERATION FEEDBACKS}

\section{A. Contact Transition Control Incorporating Only Velocity Feedback}

The dynamics of a single-link robot (Fig. 1) can be modeled as

$$
J \ddot{\theta}+\tau_{e}+\tau_{n}=\tau_{a}
$$

where $J, \ddot{\theta}, \tau_{n}, \tau_{a}$ and $\tau_{e}$ represent the link inertia, joint acceleration, disturbances, actuated torque, and reactive torque caused by the environment, respectively. The reactive torque $\tau_{e}$ is equal to the reactive force $\left(f_{e}\right)$ over the link length $(l)$, while the reactive force is determined by

$$
f_{e}= \begin{cases}0, & x<0 \\ B_{e} \dot{x}+k_{e} x, & x \geq 0\end{cases}
$$

where $k_{e}$ and $B_{e}$ represent the stiffness and damping factor of the contacted environment, respectively, and the variable $x$ represents the displacement of the environment surface. The reactive dynamics above is discontinuous at point $x=0$ when the approaching speed $(\dot{x})$ does not vanish and the kinetic energy thus generated needs to dissipate before a stable contact can be established. If an active force control law fails in absorbing the initial energy efficiently, oscillations and even instability might occur during the contact transition period. The oscillations are decided by the resonance of the open-loop frequency response of the force control, which can be obtained by substituting (2) into (1) as

$$
\frac{f_{f}(S)}{\tau_{a}(S)}=\frac{k_{s}\left(B_{e} S+k_{e}\right)}{l\left(J S^{2}+B_{e} S+k_{e}\right)}
$$

in which $f_{f}\left(=k_{s} f_{e}\right)$ represents the sensed force and $k_{s}$ stands for the gain of the force sensor. Thus, the frequency response varies with environmental characteristics and a force control needs to adjust its parameters in order to suit the various contacted environments.

When a robot impacts an environment capable of consuming less kinetic energy, a conventional closed-loop force control is unable to suppress the oscillations generated. To damp out the oscillations, a velocity feedback term may be introduced and supplemented to the force control law (for example, the proportional law)

$$
\tau_{a}=k_{f} l\left(f_{d}-f_{f}\right)-k_{v} * \dot{\theta}
$$

where $f_{d}$ represents the desired force, and $k_{v}$ and $k_{f}$ denote the velocity feedback and the force control law, respectively. Suppose that the robot impacts the environmental surface with a nonzero approaching velocity $\dot{\theta}_{0}$ at time $t=0$, the system kinetic energy at any time $t>0$ is governed by

$$
E_{i}(0)+E_{a}(t)=E_{c}(t)+E_{r}(t)
$$

where $E_{i}$ is the initial kinetic energy, and $E_{a}, E_{c}$, and $E_{r}$ are the actuating work, the work done by the reaction force, and the kinetic energy at time $t$, respectively. They are determined by

$$
\left\{\begin{array}{l}
E_{i}=\frac{1}{2} J \dot{\theta}_{0}^{2} \\
E_{a}=\int_{0}^{t} \tau_{a}(t) \dot{\theta}(t) d t \\
E_{c}=\int_{0}^{t} l f_{e}(t) \dot{\theta}(t) d t \\
E_{r}=\frac{1}{2} J \dot{\theta}^{2}(t) .
\end{array}\right.
$$


Assuming $f_{d}$ is constant and the initial velocity $\dot{\theta}_{0}$ vanishes at point $x=x_{e}$ at time $t=t_{e}$, and substituting (4) and (6) into (5) yield

$$
\left\{\begin{array}{c}
\frac{1}{2} J \dot{\theta}^{2}=\frac{1}{2} J \dot{\theta}_{0}^{2}+k_{f} f_{d} x(t)-\frac{1}{2} k_{s} k_{f} k_{e} x^{2}(t) \\
\quad-\tilde{E}_{k e}-\tilde{E}_{b e}-\tilde{E}_{v} \\
\tilde{E}_{k e}=\frac{1}{2} k_{e} x^{2}(t), \tilde{E}_{b e}=\left(k_{s} k_{f}+1\right) l^{2} B_{e} \hat{x}(t), \\
\tilde{E}_{v}=k_{v} \hat{x}(t), \quad \text { when } 0<x \leq x_{e}, 0<t \leq t_{e} \\
\hat{x}=\int_{0}^{t}(\dot{\theta})^{2} d t>0
\end{array}\right.
$$

in which $\tilde{E}_{k e}$ is the potential energy possessed by the environment, $\tilde{E}_{b e}$ is the energy dissipated by the environment, and $\tilde{E}_{v}$ is the energy consumed by the velocity feedback. It is obvious that the velocity feedback plays a role similar to the damping factor $B_{e}$, in terms of dissipation of the initial kinetic energy generated by the nonzero impact speed. Theoretically, any initial energy might be dissipated in the period of time $\left[0, t_{e}\right]$ by increasing $k_{v}$ and an overdamped contact transition might be held without any oscillations. However, the gain $k_{v}$ is generally frequency weighted, and restricted by high-frequency uncertainties and sensor noises in order to maintain the closed-loop stability. Hence, the velocity feedback has a limited bandwidth of $\omega_{c v}$, which confines the ability of $\tilde{E}_{v}$ responding to the force oscillations at high frequency.

Supposing that the oscillation frequency $\omega_{r}$ during the contact transition have been determined by (3), $\tilde{E}_{v}$ can be expressed by

$$
\tilde{E}_{v}\left(\omega_{r}\right)=\left|k_{v}\left(j \omega_{r}\right)\right| \hat{x} \approx \begin{cases}\left|k_{v}(j 0)\right| \hat{x}, & \text { when } \omega_{r} \leq \omega_{c v} \\ 0, & \text { when } \omega_{r}>\omega_{c v}\end{cases}
$$

where $\left|k_{v}\left(j \omega_{r}\right)\right|$ is the gain of the velocity feedback control at the frequency $\omega_{r}$, and $\omega_{c r}$ represents the bandwidth of $k_{v}(j \omega)$. Thus, the velocity feedback loop as an energy consumer is of little use when the oscillation frequency $\omega_{r}$ exceeds the bandwidth $\omega_{c v}$ considerably, while it is effective when the oscillation frequency is much smaller than $\omega_{c v}$, i.e., when the environment is sufficiently elastic.

\section{B. Contact Transition Incorporating Both Velocity and Acceleration Feedback}

To enhance the control performance during the stiff contact transition, a linear accelerometer (Fig. 1) is implemented and its output is also used as the feedback information, i.e.,

$$
\tau_{a}=k_{f} l\left(f_{d}-f_{f}\right)-k_{a} \int_{0}^{t}\left(\ddot{\theta}+k_{v} \dot{\theta}\right) d t
$$

where $k_{a}$ is the gain of the acceleration feedback control. Substituting this control law into (5) and (6) yields the energy-consuming term due to the acceleration and velocity feedbacks

$$
\tilde{E}_{a v}=k_{a} \hat{x}+k_{v} k_{a} x^{2} /\left(2 l^{2}\right)
$$

in which

$$
\hat{x}=\int_{0}^{t}(\dot{\theta})^{2} d t .
$$

For the oscillations of frequency $\omega_{r}$, the energy consumer $\tilde{E}_{a v}$ can be further expressed as

$$
\tilde{E}_{a v}\left(\omega_{r}\right)=\left|k_{a}\left(j \omega_{r}\right)\right| \hat{x}+\left|k_{a}\left(j \omega_{r}\right)\right|\left|k_{v}\left(j \omega_{r}\right)\right| x^{2} /\left(2 l^{2}\right) .
$$

Comparing (10) and (7) shows that the gain $k_{a}$ plays a role similar to the velocity feedback $k_{v}$. However, the bandwidth of the acceleration feedback can be raised much higher than that of the velocity feedback, i.e.,

$$
\omega_{c a} \gg \omega_{c v}
$$

where $\omega_{c a}$ denotes the bandwidth of $k_{a}(j \omega)$ and, therefore, $\tilde{E}_{a v}$ can be approximated by

$$
\tilde{E}_{a v}\left(\omega_{r}\right) \approx\left\{\begin{array}{l}
\left|k_{a}(j 0)\right| \hat{x}+\left|k_{a}(j 0)\right|\left|k_{v}(j 0)\right| x^{2} /\left(2 l^{2}\right), \\
\text { when } \omega_{r} \leq \omega_{c v} \\
\left|k_{a}(j 0)\right| \hat{x}, \quad \text { when } \omega_{c v}<\omega_{r} \leq \omega_{c a} .
\end{array}\right.
$$

By comparison of (8) and (14), it can be seen that the introduction of the acceleration feedback loop enhances the ability of the robot in suppressing the contact oscillations when $\omega_{r} \leq \omega_{c v}$, and makes it possible to damp the oscillation in a higher bandwidth $\omega_{c v}<\omega_{r} \leq \omega_{c a}$. It should also be noted that (13) is practically reasonable due to the high bandwidth of the linear accelerometer and the low-pass filtering capability of the integral force control law.

Fig. 2 shows the unified scheme proposed for free-motion, contact transition and postcontact force tracking control, where the force controller $G_{f}(s)$ is placed in parallel to the state feedbacks $G_{a}(s), G_{v}(s)$, and $G_{p}(s) . K_{1}, K_{2}$, and $K_{3}$ are controllable switches. The switch $K_{1}$ is off, and the switches $K_{2}$ and $K_{3}$ are on during the phase of free motion, i.e., when $f_{f}<\varepsilon$, where $\varepsilon$ is a prespecified positive small constant. In this situation, the scheme is an independent joint controller with joint acceleration feedback to resist the torque disturbance $\tau_{n}$. The switches $K_{1}$ and $K_{3}$ are on, and the switch $K_{2}$ is off when $f_{f} \geq \varepsilon$, the control scheme is changed to the contact transition and force control mode, which is composed of the force, velocity, and acceleration feedbacks.

It should be noted that the contact transition/force tracking control mode should be activated as soon as the contact occurs, namely, $f_{f}>\varepsilon=0$. In this paper, a small constant $\varepsilon=10 \%$ desired force is assumed as a "zero margin" ( $\varepsilon=0)$ is practically infeasible.

\section{Postcontact Force Tracking Performance}

In the preceding section, the contact transition was analyzed under the proportional force control law enhanced by either the velocity feedback (4) or both the velocity and acceleration feedbacks (9). As a matter of fact, for the force tracking control during the postcontact period, an integral law is more effective for both soft and hard environments in contact [16], but it has poor contact transition performance [9]. In this paper, a simple 


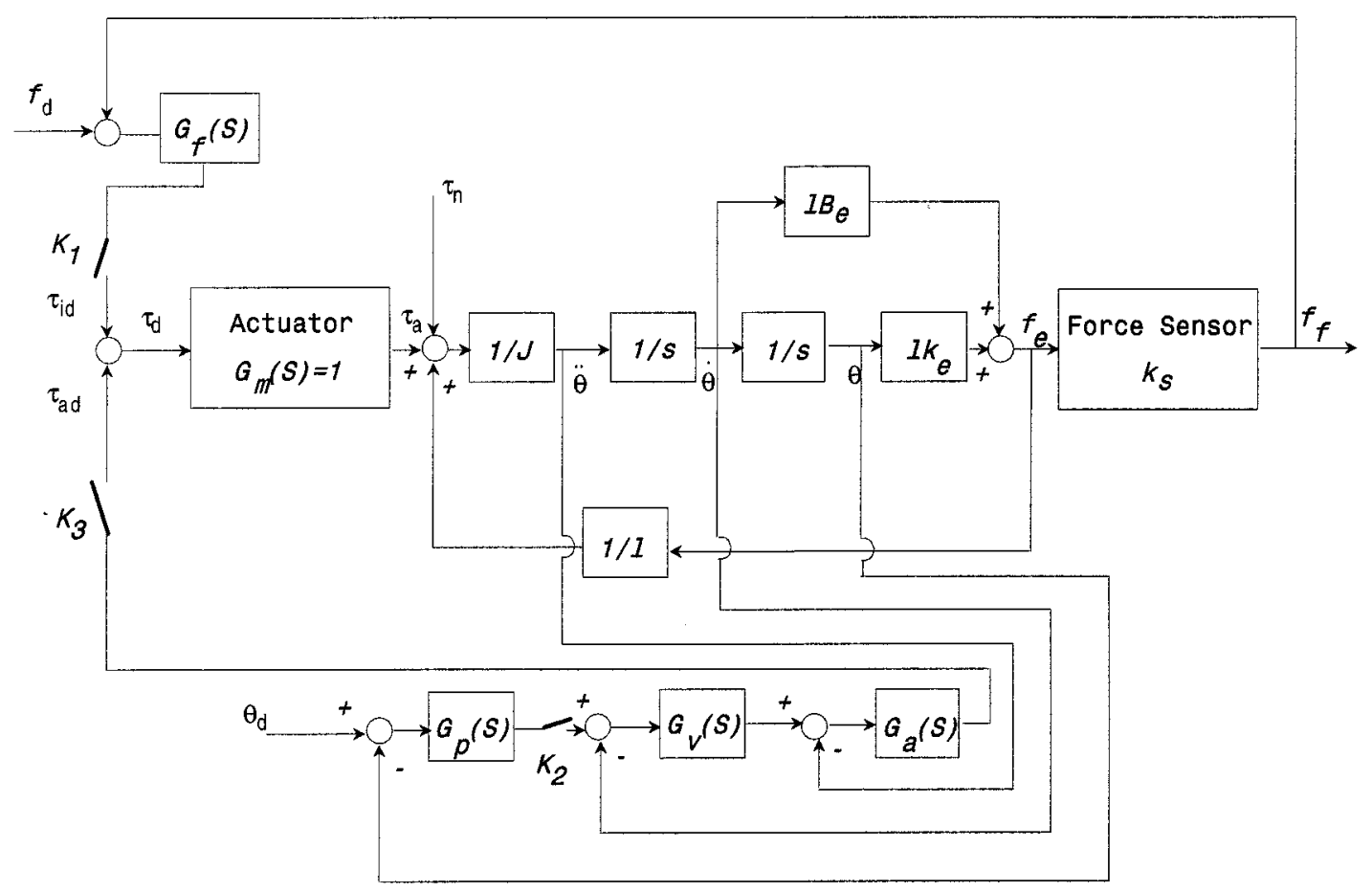

Fig. 2. Proposed contact transition control.

unified integral law (9) is employed for both the contact transition and postcontact force tracking. The control laws in Fig. 2 are chosen as follows:

$$
G_{f}(s)=\frac{k_{f}}{s} \quad G_{p}(s)=k_{p} \quad G_{v}(s)=k_{v} \quad G_{a}(s)=\frac{k_{a}}{s}
$$

where $k_{f}, k_{p}, k_{v}$, and $k_{a}$ are constants. The closed-loop transfer function during the postcontact (force tracking) period is, thus, obtained as

$$
f_{f}(s)=G_{f c}(s) f_{d}(s)+G_{\tau c}(s) \tau_{n}(s)
$$

where

$G_{f c}(s)=\frac{k_{f}\left(B_{e} s+k_{e}\right)}{J s^{3}+\left(k_{a}+B_{e}\right) s^{2}+\left(k_{v} k_{a}+k_{f} B_{e}+k_{e}\right) s+k_{f} k_{e}}$

$G_{\tau c}(s)=\frac{s\left(B_{e} s+k_{e}\right)}{J s^{3}+\left(k_{a}+B_{e}\right) s^{2}+\left(k_{v} k_{a}+k_{f} B_{e}+k_{e}\right) s+k_{f} k_{e}}$.

According to the Routh's stability criterion [17], the closed-loop stability requires

$$
\begin{array}{r}
0<k_{f}<\frac{\left(k_{v} k_{a}+k_{e}\right)\left(k_{a}+B_{e}\right)}{\left(J k_{e}-B_{e}^{2}-B_{e} k_{a}\right)}, \\
\text { when } J>\frac{B_{e}\left(B_{e}+k_{a}\right)}{k_{e}} .
\end{array}
$$

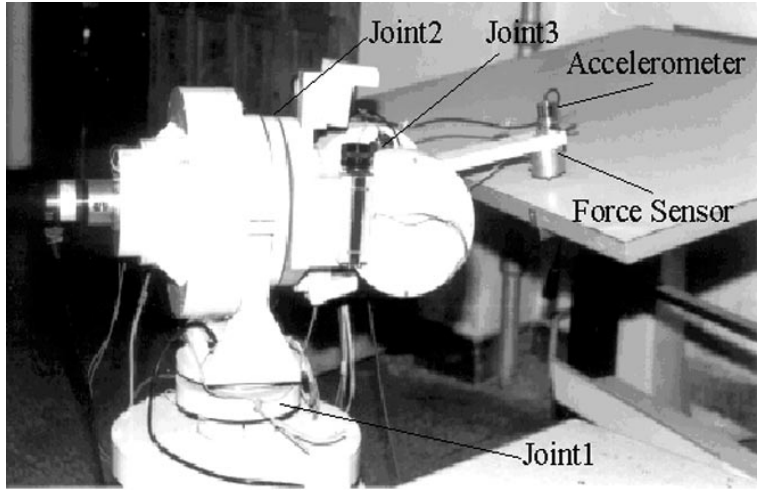

Fig. 3. Three-link direct-drive robot.

If no velocity and acceleration feedbacks are applied in the postcontact period, the closed-loop transfer function and stability conditions are given by

$$
f_{f}(s)=\bar{G}_{f c}(s) f_{d}(s)+\bar{G}_{\tau c}(s) \tau_{n}(s)
$$

where

$$
\begin{aligned}
& \bar{G}_{f c}(s)=\frac{k_{f}\left(B_{e} s+k_{e}\right)}{J s^{3}+B_{e} s^{2}+\left(k_{e}+k_{f} B_{e}\right) s+k_{f} k_{e}} \\
& \bar{G}_{\tau c}(s)=\frac{s\left(B_{e} s+k_{e}\right)}{J s^{3}+B_{e} s^{2}+\left(k_{e}+k_{f} B_{e}\right) s+k_{f} k_{e}}
\end{aligned}
$$

and

$$
0<k_{f}<\frac{k_{e} B_{e}}{J k_{e}-B_{e}^{2}}, \quad \text { when } J>\frac{B_{e}^{2}}{k_{e}} .
$$

It can be seen from (16) to (18) that there is no steady-state error between the actual contact force $\left(f_{f}\right)$ and the desired force 


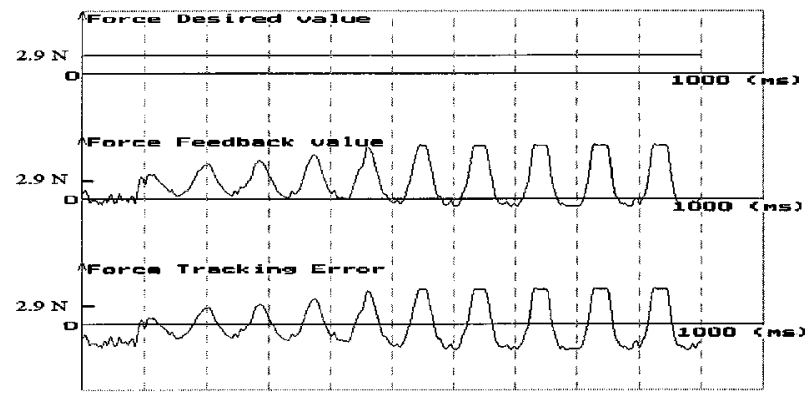

(a)

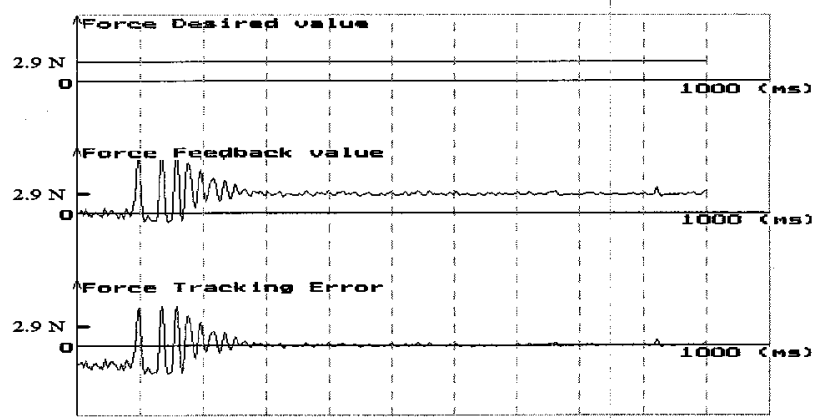

(b)

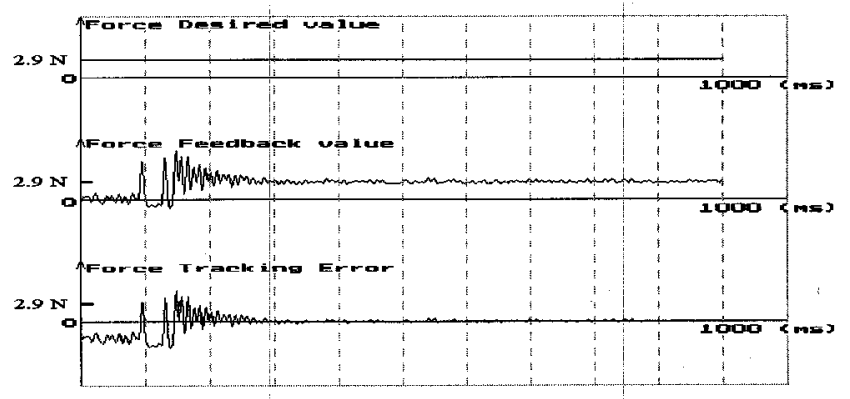

(c)

Fig. 4. Transition control under only integral force control. (a) Elastic (sponge) environment, (b) Less elastic (cardboard) environment. (c) Hard (steel plate) environment.

$\left(f_{d}\right)$. By comparing (16)-(18) with (20)-(22), it is found that the gains $k_{a}$ and $k_{v}$ supplement the coefficients of the denominator terms $s$ and $s^{2}$, and hence enhance the ability of the closed-loop system in damping the oscillation in the desired force $\left(f_{d}\right)$ and resisting the force disturbances $\tau_{n}$. Moreover, comparing (19) with (23) shows that the introduction of $k_{a}$ also expands the stable upper bound of $k_{f}$.

\section{EXPERIMENTS}

The experiment is carried out on the third joint of a three-link direct-drive robot (Fig. 3). A one-dimensional force sensor and a linear accelerometer are implemented at the end of the last link, and other sensors equipped include the current sensors, tachometers, and encoders. The accelerometer used has a sensitive band $\left(0-200 \mathrm{~Hz}\right.$ ) and sensitivity of $10^{-5} \mathrm{~g}$ (the gravity acceleration), while the force sensor used has inherent resonant frequency of $20 \mathrm{kHz}$ and sensitivity of $1 \mathrm{mv} / \mathrm{v}$. The force closed loop is built on a Pentium 100 personal computer at a sampling rate of $1 \mathrm{kHz}$. With respect to elastic (sponge), less elastic (cardboard), and hard (steel plate) contact surfaces, the closed-loop transition control without velocity and acceleration feedbacks, with only velocity feedback, and with both velocity and acceleration feedbacks are investigated, respectively. The postcontact force tracking performance of the closed-loop system incorporating the velocity and acceleration feedbacks are also investigated experimentally.

\section{A. Contact Transitions with Integral Force Control Only}

During the phase of free motion, the switch $K_{1}$ is off and, $K 2$ and $K 3$ are on, as shown in Fig. 2. The control becomes an independent joint controller only with the acceleration feedback. The approaching speed at the end of the link is $0.195 \mathrm{~m} / \mathrm{s}$, i.e., the joint speed $36^{\circ} / \mathrm{s}$ equivalently, for all three contact surfaces. Once $f_{f}>\varepsilon$, the switch $K_{1}$ is switched on and $K_{3}$ off. Thus, the control structure is changed into the transition control mode without velocity and acceleration feedbacks. In the experiment, the desired contact force $f_{d}$ and the force margin $\varepsilon$ are set as 2.9 and $0.3 \mathrm{~N}$, respectively, and the control laws chosen are

$$
\begin{aligned}
& G_{f}(s)=48.0 / s \\
& G_{a}(s)=3.96 \times 10^{6} /[(s+30)(s+219)] \\
& G_{v}(s)=4100 /(s+75) .
\end{aligned}
$$

Fig. 4(a)-(c) shows the desired force, actual force, and force tracking error during the transition from free motion to constrained motion, with respect to the elastic, less elastic, and hard environments. It is shown that the system is unstable for the elastic surface, with oscillations of approximate resonant frequency $14.9 \mathrm{~Hz}$, while the contact transitions are eventually stabilized for the less elastic and hard surfaces, due to the higher resonant frequencies. However, there still exist oscillations of high frequencies of about 73 and $100 \mathrm{~Hz}$ in the initial phase of transition. This experiment has shown that the integral force control law usually leads to an unstable closed-loop system when the environment in contact is elastic. 


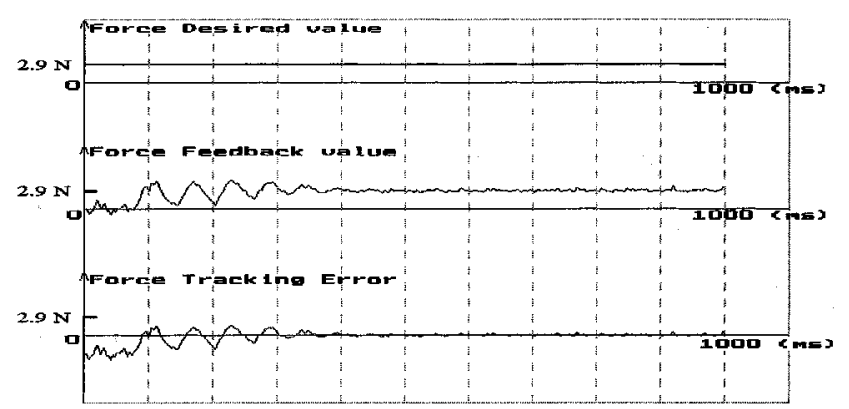

(a)

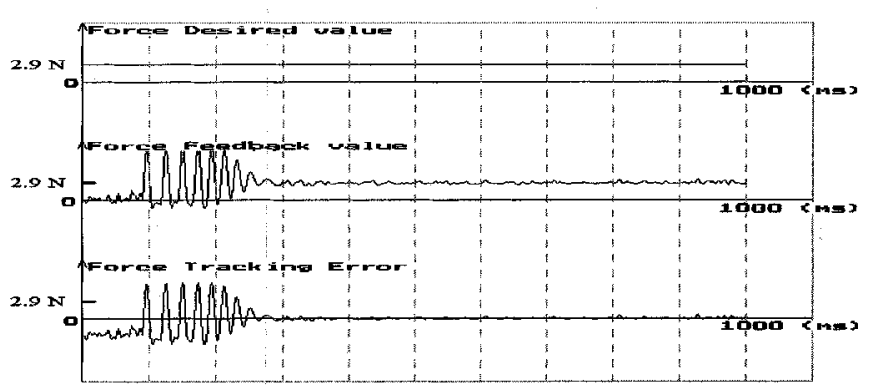

(b)

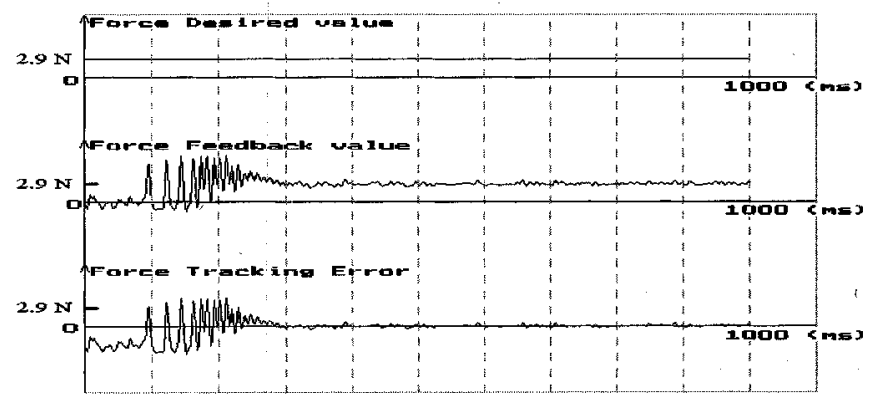

(c)

Fig. 5. Contact transition control with only joint velocity feedback. (a) Elastic (sponge) environment. (b) Less elastic (cardboard) environment. (c) Hard (steel plate) environment.

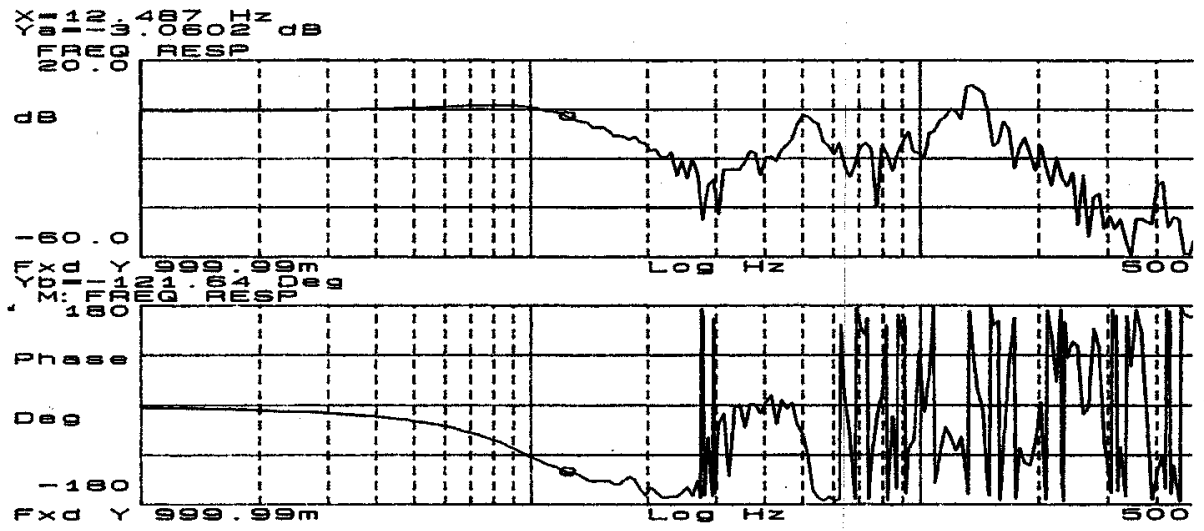

Fig. 6. Closed-loop frequency response of velocity feedback control.

\section{B. Contact Transition Control with Velocity Feedback Only}

In this experiment, $G_{v}(s)$ is connected directly to $\tau_{a d}$, as shown in Fig. 3, while the acceleration feedback is inactivated. The control laws are given in (24). During the phase of free motion, the switch $K_{1}$ is off and, $K_{2}$ and $K_{3}$ are on, while the switches $K_{1}$ and $K_{3}$ are on, and $K_{2}$ is off once the contact is established. The approaching speed is still $0.195 \mathrm{~m} / \mathrm{s}$ in this case. Fig. 5(a)-(c) shows the desired force, actual force, and force tracking error for the elastic, less elastic, and hard environments, respectively. It is found that the velocity feedback can damp low-frequency oscillations significantly and lead to a stable transition when the robot is in contact with the elastic environment, by comparison with the results given in Fig. 4(a).
However, the velocity feedback has little effect on attenuating high-frequency oscillations in the contact transition, as shown in Fig. 5(a) and (b). The above results are also verified by the closed-loop frequency response of the velocity feedback control shown in Fig. 6, whose 3-dB bandwidth is only $12.5 \mathrm{~Hz}$.

\section{Contact Transition Control with Both Joint Acceleration and Velocity Feedbacks}

In this experimental investigation, all initial conditions and switch states are the same as those in the preceding section. Fig. 7(a)-(c) shows the desired force, actual force, and force tracking error for the elastic, less elastic, and hard environments, 


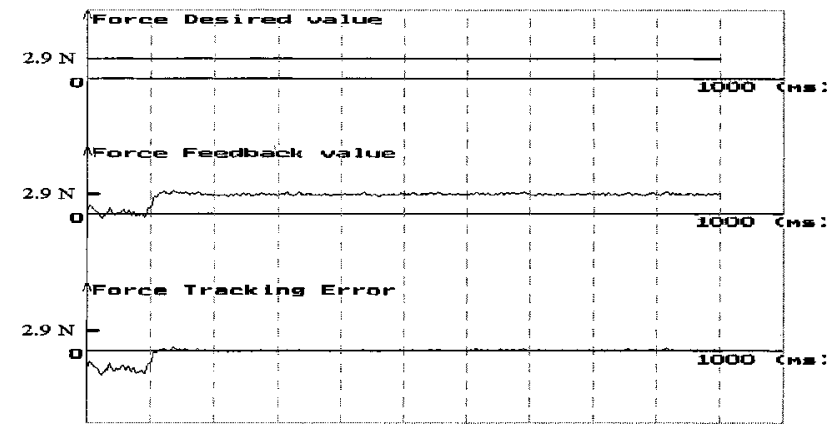

(a)

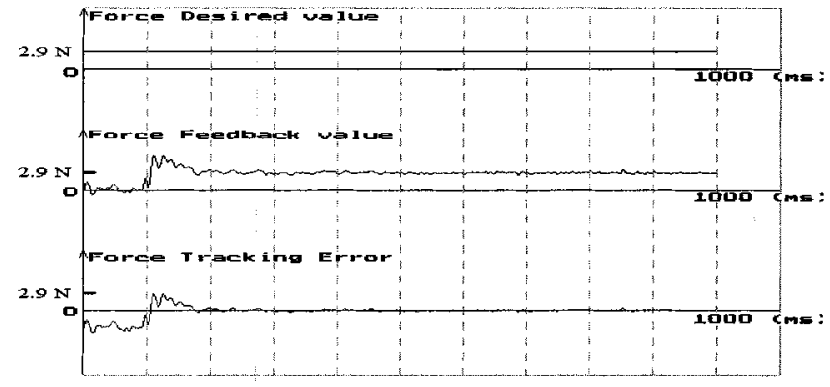

(b)

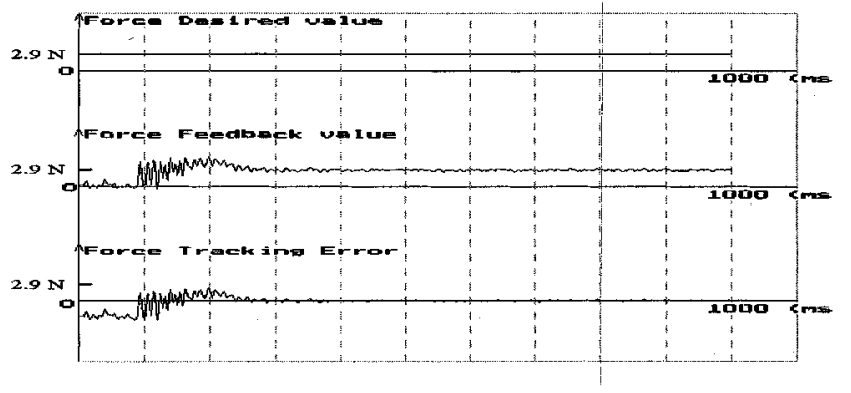

(c)

Fig. 7. Contact transition control with both joint acceleration and velocity feedbacks. (a) Elastic (sponge) environment. (b) Less elastic (cardboard) environment. (c) Hard (steel plate) environment.

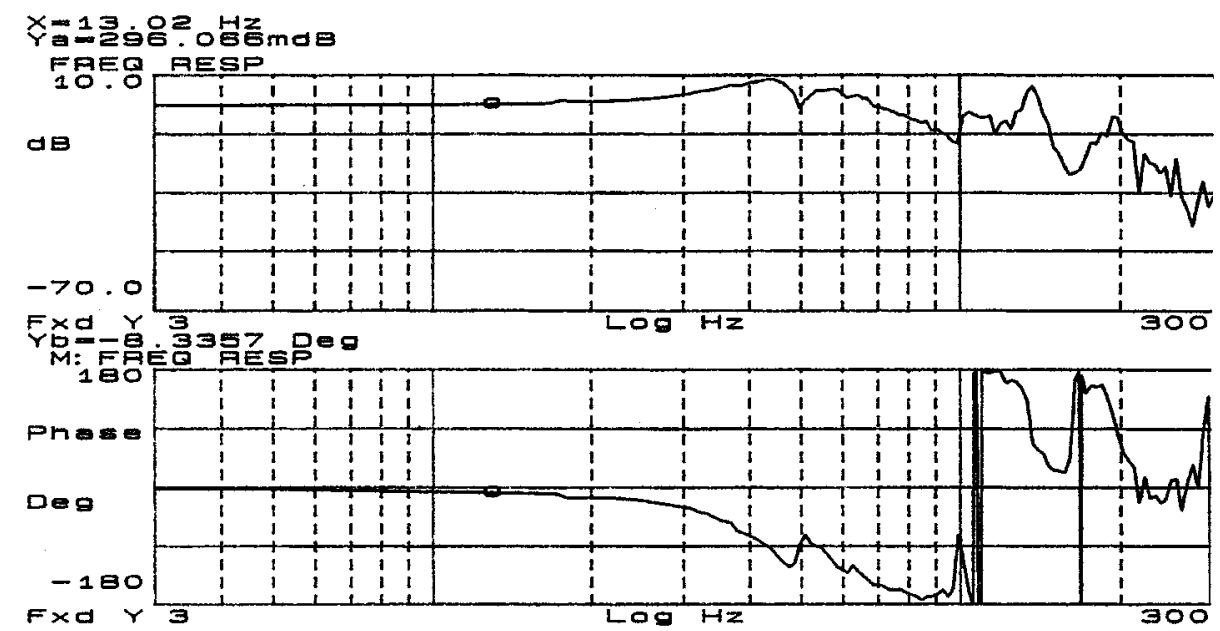

Fig. 8. Closed-loop frequency response of acceleration feedback control.

respectively, when both joint acceleration and velocity feedbacks are employed. Comparing Fig. 7 with Fig. 5 shows that the oscillations in the elastic and less elastic contact transitions are damped almost completely and the contact transitions are more stable in this case, while the oscillations in the hard contact transition are also reduced substantially. As can also be seen from Figs. 6 and 8, the closed-loop bandwidth of the acceleration feedback control is as high as seven times that of the velocity feedback control. Thus, it can be concluded from the above results that the joint acceleration feedback helps resist high-frequency oscillations and thus enables the simple integral force control law robust enough to achieve nearly identical performance for different environmental surfaces.

\section{Postcontact Force Tracking Performance}

With respect to the contact control incorporating the velocity and acceleration feedbacks, the postcontact (force tracking) starts in about $100 \mathrm{~ms}$ after the impact, as shown in Fig. 7. To demonstrate how well the closed-loop system responds to an abrupt change in the desired force in the postcontact phase, a force pulse lasting $50 \mathrm{~ms}$ is added to the constant desired force, and the force response of the system is then measured by the 


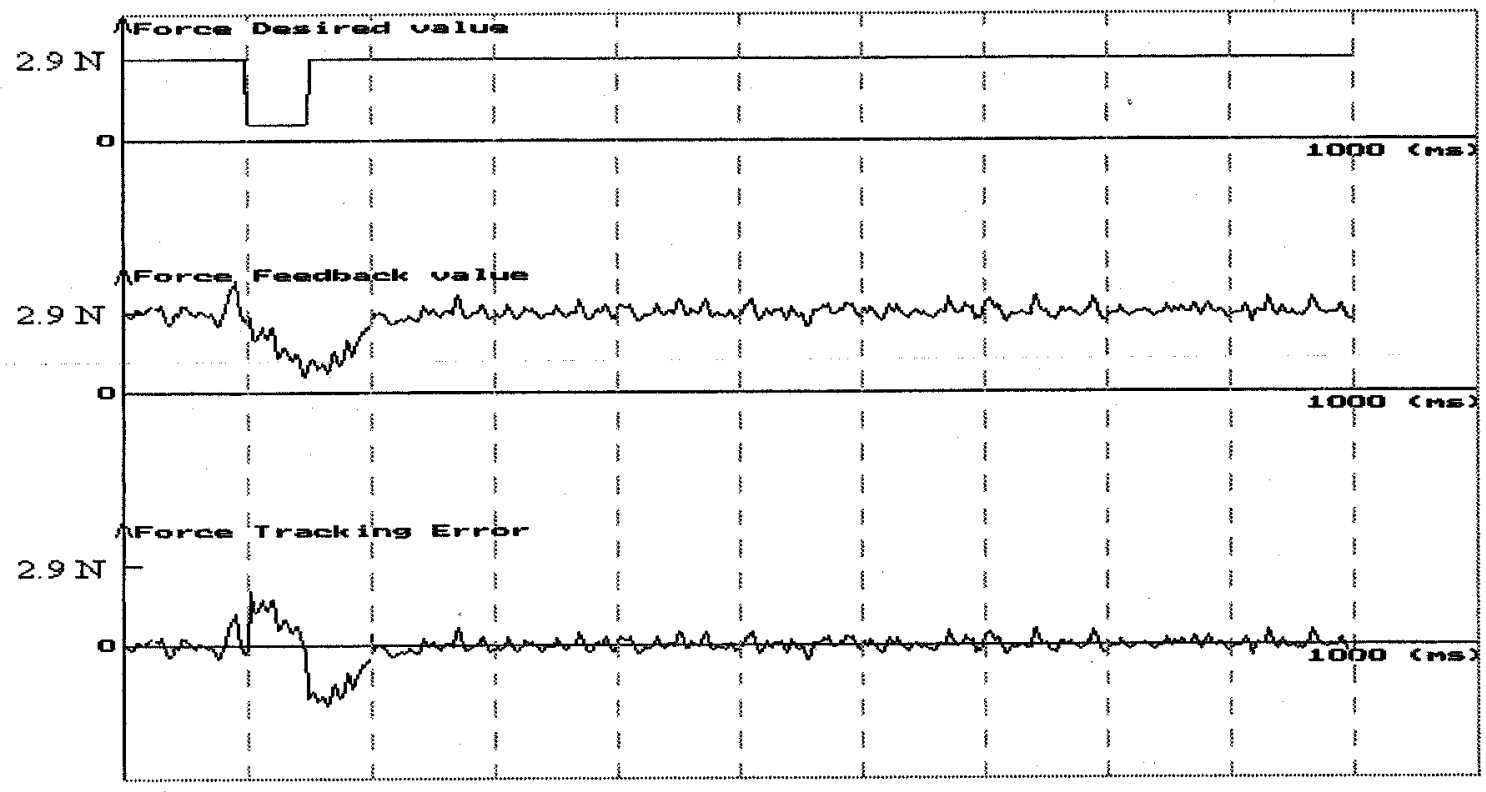

(a)

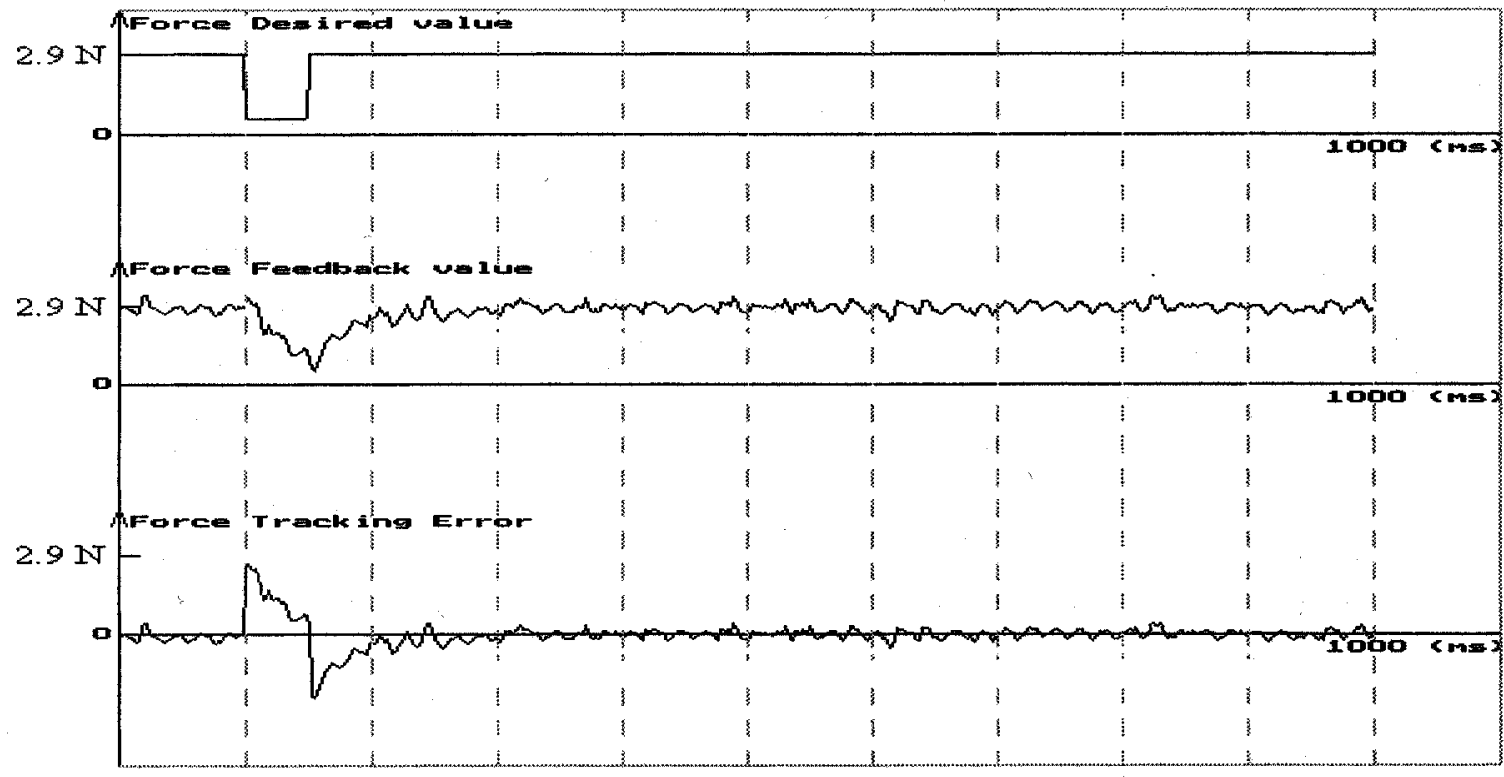

(b)

Fig. 9. Force response of the system to an abrupt change (with velocity feedback only). (a) Elastic (sponge) environment. (b) Hard (steel plate) environment.

force sensor. Figs. 9 and 10 show the force responses of the control system with the velocity feedback only, and both the velocity and acceleration feedbacks, respectively. It is shown that the disturbance in the desired force is suppressed almost completely in the postcontact phase, when the force control is enhanced by both the acceleration and velocity feedbacks. However, if such an abrupt change in the desired force is regarded as the force trajectory needed to be tracked, the acceleration feedback will degrade the tracking performance during the postcontact period. Thus, there should be a tradeoff between the disturbance damping and the force tracking performance.

\section{CONCLUSION}

A classical integral force control scheme has been enhanced for controlling the contact transition by means of joint acceleration and velocity feedbacks. The feedback loops serve as a robust damper to suppress the contact oscillations. A unified control structure is proposed for free motion, contact transition, and postcontact control. The influence of the acceleration and velocity feedbacks on the force tracking performance during the postcontact period was discussed. Extensive experiments were carried out, including the sole force control, the force 


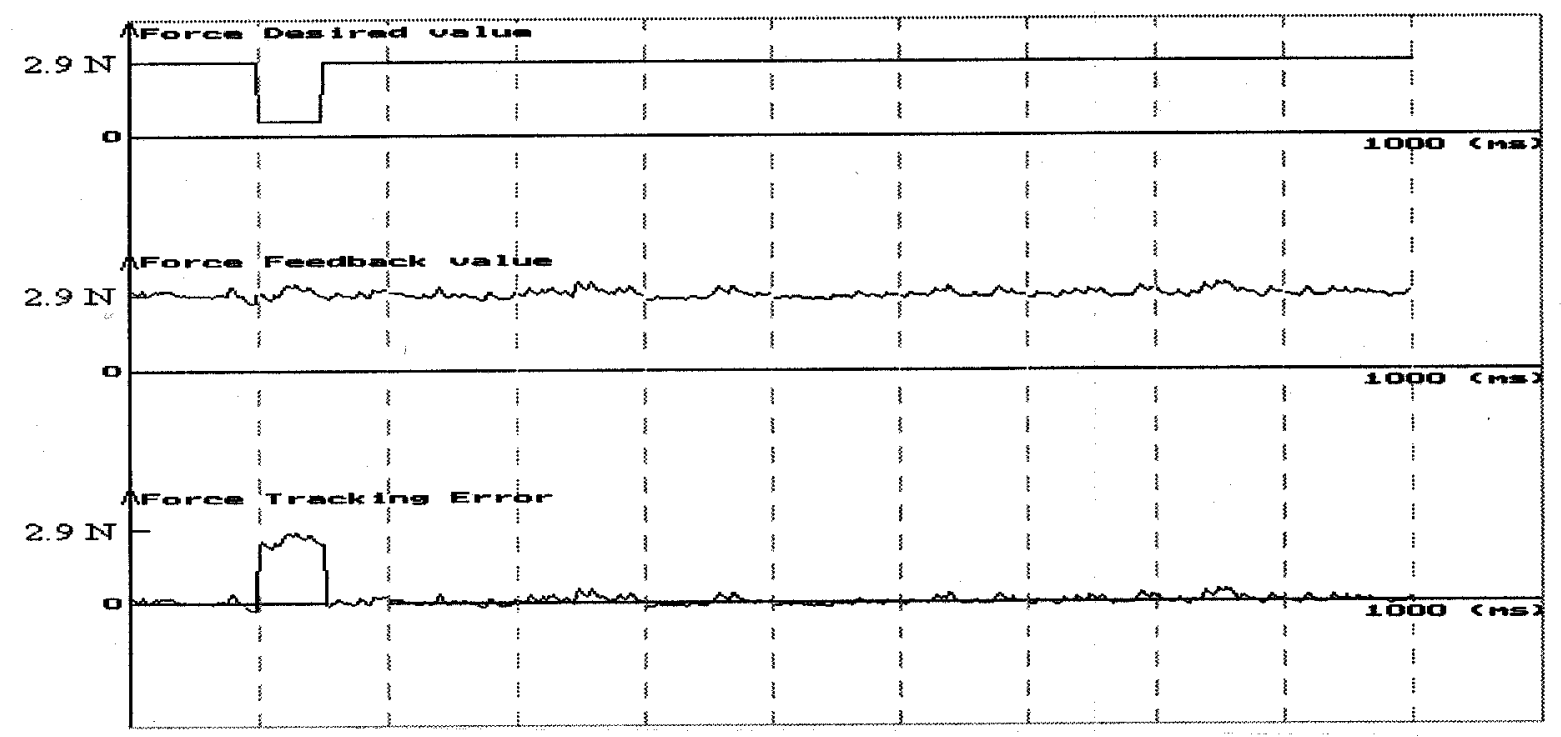

(a)

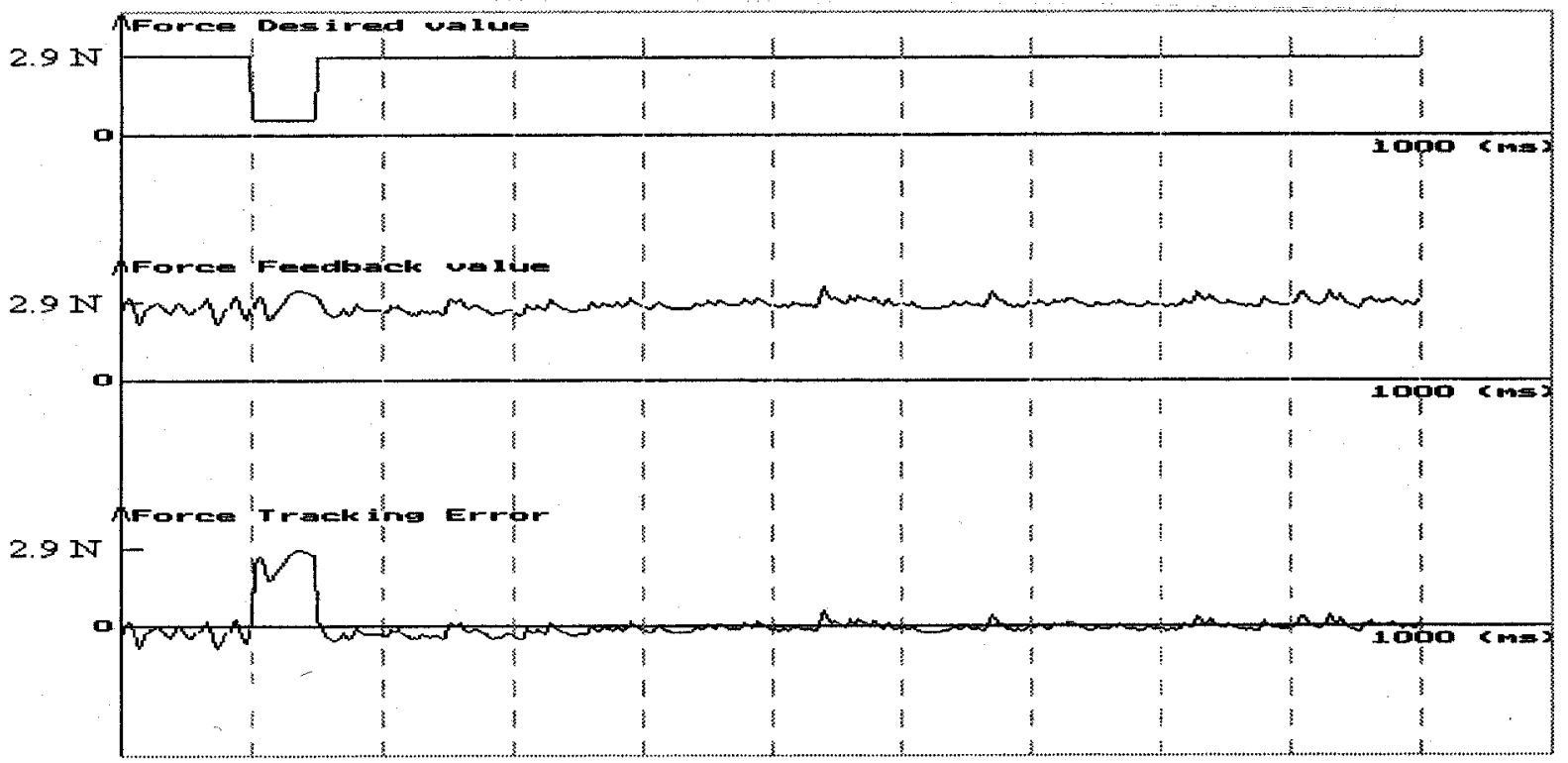

(b)

Fig. 10. Force response of the system to an abrupt change (with both acceleration and velocity feedbacks). (a) Elastic (sponge) environment. (b) Hard (steel plate) environment.

control plus the velocity feedback, and the force control plus both the velocity and acceleration feedbacks. It is shown by experiments that the force control enhanced by the joint acceleration feedback, due to its high bandwidth, can help damp out the oscillations substantially in the contact transition phase, under the environments of various stiffnesses. However, since the joint acceleration feedback degrades the force tracking performance during the postcontact period, a tradeoff should be made between the oscillation damping performance and the force tracking ability when designing the control system for contact tasks.

\section{REFERENCES}

[1] J. K. Mills, "Stability of robotic manipulators during transition to and from compliant motion," Automatica, vol. 26, no. 5, pp. 861-874, Aug. 1990.

[2] J. K. Mills and D. M. Lokhorst, "Experimental results in manipulators contact task control," in Proc. IEEE Int. Conf. Robotics and Automation, Apr. 1991, pp. 1645-1651.

[3] — "Control of robotic manipulators during general task execution: A discontinuous control approach," Int. J. Robot. Res., vol. 12, no. 2, pp. 146-163, Apr. 1993.

[4] R. Volpe and P. Khosla, "A theoretical and experimental investigation of impact control for manipulators," Int. J. Robot. Res., vol. 12, no. 4, pp. 351-365, Aug. 1993. 
[5] N. Hogan, "Stable execution of contact tasks using impedence control," in Proc. IEEE Int. Conf. Robotics and Automation, Mar. 30-Apr. 3, 1987, pp. 1047-1054.

[6] R. Vossoughi and M. Donath, "Robot hand impedance control in the presence of mechanical nonlinearities," in Proc. ASME Winter Annu. Meeting, Miami, FL, Nov. 17-22, 1985, pp. 120-128.

[7] H. Sereji, D. Lim, and R. Steele, "Experiment in contact control," $J$. Robot. Syst., vol. 13, no. 2, pp. 53-57, Feb. 1996.

[8] H. Seraji and R. Colbaugh, "Force tracking in impedance control," Int. J. Robot. Res., vol. 16, no. 1, pp. 97-117, Feb. 1997.

[9] K. Youcef-Toumi and D. A. Gutz, "Impact and force control," in Proc. IEEE Int. Conf. Robotics and Automation, 1989, pp. 410-416.

[10] O. Khatib and J. Burdick, "Motion and force control of robot manipulators," in Proc. IEEE Int. Conf. Robotics and Automation, Apr. 7-10, 1986, pp. 1381-1386.

[11] H. P. Qian and J. De Schutter, "Introducing active linear and nonlinear damping to enable high gain force control in case of stiff contact," in Proc. IEEE Int. Conf. Robotics and Automation, Nice, France, May 12-14, 1992, pp. 1374-1379.

[12] J. M. Hyde and R. Cutkosky, "Controlling contact transition," IEEE Contr. Syst. Mag., vol. 14, no. 1, pp. 25-30, Feb. 1994.

[13] _ - "Contact transition control: An experimental study," in Proc. IEEE Int. Conf. Robotics and Automation, Atlanta, GA, May 2-6, 1993, pp. 363-368.

[14] M. Vukobratovic, "How to control robots interacting with dynamic environment," J. Intell. Robot. Syst., vol. 19, no. 2, pp. 119-152, Dec. 1997.

[15] S. W. Weng and K. Y. Young, "An impact control scheme inspired by human reflex," J. Robot. Syst., vol. 13, no. 12, pp. 837-855, Dec. 1996.

[16] N. Mandal and S. Payandeh, "Control strategies for robotics contact tasks," J. Robot. Syst., vol. 12, no. 1, pp. 67-92, Feb. 1995.

[17] K. Ogata, Modern Control Engineering, 3rd ed. Englewood Cliffs, NJ: Prentice-Hall, 1997, pp. 232-234.

[18] G. J. Liu and A. A. Goldenberg, "Robust hybrid impedance control of robot manipulator via a tracking control method," in Proc. EEE/RSJ/GI Int. Conf. Intelligent Robots and Systems, 1994, pp. 1594-1601.

[19] T. J. Tarn, Y. Y. Wu, N. Xi, and A. Isidori, "Force regulation and contact transition control," IEEE Contr. Syst. Mag., vol. 16, pp. 32-40, Feb. 1996.

[20] Y. Y. Wu, T. J. Tarn, N. Xi, and A. Isidori, "On robust impact control via positive acceleration feedback for robot manipulators," in Proc. IEEE Int. Conf. Robotics and Automation, 1996, pp. 1891-1896.

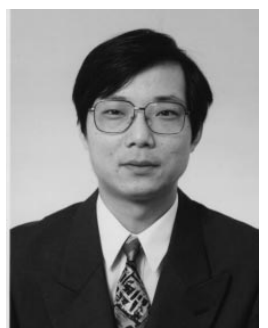

W. L. Xu (M'95-SM'99) received the Ph.D. degree in mechatronic engineering from Beijing University of Aeronautics and Astronautics, Beijing, China, in 1988.

He was with Southeast University, China, University of Stuttgart, Germany, Ritsumeikan University, Japan, and the City University of Hong Kong. $\mathrm{He}$ is currently a Senior Lecturer (Mechatronics and Automation) in the Institute of Technology and Engineering, Massey University, Palmerston North, New Zealand. His research interests include intelligent mobile robots, service robots and automation, sensor-based flexible manipulators, and computational intelligence. He is also Principal Investigator of a number of funded and contracted projects in robotics and service automation

Dr. Xu is an active member of the New York Academy of Sciences and a senior member of the Chinese Society of Mechanical Engineering.

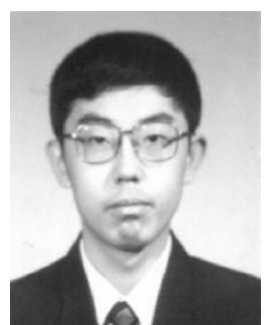

J. D. Han received the Ph.D. degree in mechatronics from Harbin Institute of Technology, Harbin, China, in 1998.

$\mathrm{He}$ is currently an Associate Professor in the Robotics Laboratory, Shenyang Institute of Automation, Chinese Academy of Sciences, Shenyang, China. From 1998 to 1999, he was a Senior Research Assistant in the Centre for Intelligent Design, Automation and Manufacturing, City University of Hong Kong. His research interests include sensor-based robot control, flexible manipulators, intelligent mobile robots, and multirobot coordination and cooperation. He is also the Principal Investigator of several "863" projects in China.

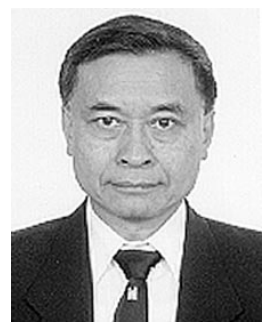

S. K. Tso (SM' 81$)$ joined the City University of Hong Kong, Hong Kong, China, as a Professor of mechatronics and automation in 1995, following a long period of service with the University of Hong Kong. He is currently Director of the Centre for Intelligent Design, Automation and Manufacturing. He has carried out research in the broad fields of industrial electronics and automation and control, supervised numerous Ph.D., M.Phil., and M.Sc. students, and authored more than 250 published refereed scientific papers. He has worked in the industrial electronics and control industries and served as a Consultant for a number of government and industrial projects. He has held Visiting Professorships at many universities, including the University of Toronto, University of Waterloo, and National University of Singapore.

Prof. Tso is a Fellow of the Institution of Electrical Engineers, U.K., and the Hong Kong Institution of Engineers. 
Experimental study of contact transition control incorporating joint acceleration feedback

$\mathrm{Xu}, \mathrm{W} . \mathrm{L}$.

2000-09

http://hdl.handle.net/10179/9602

22/04/2023 - Downloaded from MASSEY RESEARCH ONLINE 